\title{
APPLYING THE HEALTH BELIEF MODEL IN EXPLAINING THE STAGES OF EXERCISE CHANGE IN OLDER ADULTS
}

\author{
KRZYSZTOF SAS-NOWOSIELSKIIA, ANDRZEJ HADZIK'B , JOANNA GÓRNA², \\ MAŁGORZATA GRABARA ${ }^{\mathrm{IB}}$
IJerzy Kukuczka Academy of Physical Education in Katowice, Faculty of Physical Education, Department of Pedagogy and Psychology ${ }^{a}$, Department of Tourism and Health-Related Physical Activity ${ }^{b}$ 2 Jan Dlugosz University in Czestochowa, Faculty of Pedagogy, Department of Career Counselling

\author{
Mailing address: Małgorzata Grabara, Jerzy Kukuczka Academy of Physical Education, Faculty of Physical Education, \\ 72a Mikołowska Street, 40-065 Katowice, tel.: +48 32 2075169, fax: +48 32 3075200, \\ e-mail:m.grabara@awf.katowice.pl
}

\begin{abstract}
Introduction. The benefits of physical activity (PA) have been so well documented that there is no doubt about the significance of PA for personal and social health. Several theoretical models have been proposed with a view to understanding the phenomenon of PA and other health behaviours. The purpose of this study was to evaluate if and how the variables suggested in the Health Belief Model (HBM) determine physical activity stages of change in older adults. Material and methods. A total of 172 students of Universities of the Third Age aged 54 to 75 (mean $=62.89 \pm 4.83$ ) years agreed to participate in the study, filling out an anonymous survey measuring their stage of exercise change and determinants of health behaviours proposed by the HBM, including: perceived benefits of physical activity, perceived barriers to physical activity, perceived severity of diseases associated with sedentary lifestyle, perceived susceptibility to these diseases, and self-efficacy. Results. The results only partially support the hypothesis that the HBM predicts intentions and behaviours related to the physical activity of older adults. Only two variables were moderately-to-strongly related to stages of exercise change, namely perceived barriers and self-efficacy. Conclusion. Interventions aimed at informing older adults about the benefits of physical activity and the threats associated with sedentary lifestyle can be expected to have rather a weak influence on their readiness for physical activity.
\end{abstract}

Key words: physical activity, health behaviours, barriers to physical activity, benefits of physical activity

\section{Introduction}

It goes beyond any question that there are numerous benefits of physical activity (PA); the improvement of physical and mental health as well as the prevention of chronic diseases and premature death are but a few [1]. The fact that these benefits have been so well documented leaves no doubt about the significance of PA for personal and social health. Nevertheless, many people are still not active enough to meet recommendations [2]. Therefore, the promotion of PA remains a critically important task, though a very difficult one, as it is determined by various factors including demographic, psychological, social, biologi$\mathrm{cal}$, and behavioural ones as well as their mutual interactions and interdependencies [3]. To date, several theoretical models have been proposed with a view to understanding the phenomenon of physical activity and other health behaviours. The main objectives of the models were to "provide assumptions about behaviour and determine relations between key variables that are necessary to explain and predict behaviour" [3]. The Health Belief Model (HBM) constitutes one of the oldest models of health behaviour. Established in the 1950s, the model was elaborated to provide an explanation as to why certain people take actions which aim at preventing diseases while others avoid such actions [4].
Put simply, the HBM assumes that the likelihood of performing specific health behaviour is related to people's conviction that they are threatened with certain diseases, their evaluation of the severity of these diseases, and to the conviction that the target health behaviour allows averting the risk of developing the said diseases. In the original version of the model, these key variables were denominated as Perceived Susceptibility, Perceived Severity, and Perceived Benefits. Additional variables were Perceived Barriers, that is the costs or obstacles to the target behaviour perceived by the individual, Cues to Action such as media campaigns or the illness of a loved one, and the category of Modifying Factors which assume the form of demographic variables, personality traits, etc. Later, another variable was added, namely Self-efficacy, which is a construct relating to personal beliefs in having the ability to take the actions required to produce the expected results.

Having gained widespread popularity, the HBM model has been successfully used to describe a number of health behaviours, such as prophylactic examinations, weight control [5], mammography, prophylactic vaccination, food handling and nutrition [6, 7], wearing bicycle helmets [8], using tanning beds [9], and many others [10]. In light of the above, a question arises about the applicability of this model in predicting PA. If the model were to be applicable in this case, in order to 
undertake regular PA, the individual would be expected to be convinced that: (1) they may be at risk for diseases whose aetiology is associated with sedentary lifestyle (ischemic heart disease, non-insulin-dependent diabetes mellitus, etc.); (2) these diseases may have a very serious impact on their physical, social, and psychological functioning; (3) taking up a given physical activity may reduce the risk of developing the abovementioned diseases and/or lessen their negative effects; (4) the perceived benefits of PA outweigh the barriers to undertaking it; and, finally (5) the individual will be able to meet the challenges posed by undertaking a given PA.

Few studies regarding this issue have been conducted to date, and the efficacy of the model in predicting PA has not been confirmed conclusively. Perhaps, the reason for the lack of consistency among the results of the studies is the diversity of the surveyed populations - sick and/or elderly people vs. healthy and/or young people. Some authors claim that the HBM is a typical model for avoiding a disease. As such, its assumptions may be accurate for those who are directly at risk of or already affected by hypokinetic diseases, such as the elderly, yet it will not be helpful in understanding physical activity in healthy, young people $[3,11,12]$. Though it must be emphasised that this problem has not been extensively researched so far, and the body of existing literature discussing it is quite limited.

Another model, namely the trans-theoretical model (TTM), is considered to be one of the most popular contemporary models of physical activity behaviours. According to it, individuals move through several stages called stages of readiness to change, or simply stages of change, while undertaking PA [13]. The following five stages of change have been identified: (1) precontemplation (a lack of physical activity and lack of intention to be active), (2) contemplation (a lack of physical activity with an intention to undertake physical activity within half a year), (3) preparation (irregular PA), (4) action (regular PA but undertaken for no longer than half a year), and (5) maintenance (regular PA which has become a relatively well-established habit). Sometimes, the following two additional stages are proposed: termination, when the individual has no temptation to engage in the old behaviour which was changed at least five years earlier and has 100 percent self-efficacy in overcoming barriers to PA, and relapse, that is regression to inactive stages of contemplation or precontemplation. Neither of these additional stages has been tested extensively in PA research [11]. Although the TTM was integrated with other models of PA such as self-determination theory and the theory of planned behaviours, there are very few studies of TTM and HBM, especially with respect to older adults. Therefore, the main objective of this study was to determine if and how the variables suggested in the Health Belief Model might differ across PA stages of change in older adults.

\section{Material and methods}

\section{Participants}

The group examined in the study included 172 participants aged from 54 to 75 (mean $=62.89 \pm 4.83$ ) years, who were students of the Universities of the Third Age in Katowice, Częstochowa, and Lubliniec (Poland). The study sample consisted of 146 women (mean age $=62.42 \pm 4.64$ years) and 26 men (mean age $=65.87 \pm 5.22$ years $)$.

\section{Procedures and methods}

The study was approved by the Bioethics Committee for Scientific Research at the Jerzy Kukuczka Academy of Physical Education in Katowice, Poland.
The stages of exercise readiness were assessed by means of five items as proposed by Marcus, Rakowski, and Rossi (1992) with an additional item regarding relapse ("I exercised in the past, but I do not exercise now") [14]. The five items were as follows: "I currently do not exercise, and I do not intend to start exercising in the next six months" (precontemplation); "I currently do not exercise but am thinking about starting to exercise within the next six months (contemplation); "I currently exercise a little but not regularly" (preparation); "I currently exercise regularly but have only begun doing so within the past six months" (action); "I currently exercise regularly and have done so for longer than six months" (maintenance) [14].

The perceived benefits of PA were assessed according to a scale consisting of 12 statements related to the positive effects of physical activity on physical and mental health. The subjects' task was to assess their accuracy on a scale ranging from 1 (definitely false) to 4 (definitely true). Barriers to physical activity were assessed by means of a scale consisting of 13 statements illustrating the impediments to taking up physical activity. The subjects were to assess each of the situations presented according to a scale ranging from 1 (never a barrier) to 5 (very often a barrier). The perceived severity of diseases associated with sedentary lifestyle was assessed using a scale consisting of eight conditions (atherosclerosis and coronary heart disease, non-insulin dependent diabetes, colon cancer, depression, etc.) whose severity the subjects assessed using a scale ranging from 1 (not at all serious) to 5 (life-threatening), and then the risk of each disease was evaluated according to a scale ranging from 1 (very low risk) to 5 (very high risk). Self-efficacy was measured as confidence in continuing physical activity in eight different situations, such as lack of time, bad weather, bad mood, and the like, and assessed using a scale ranging from 1 (definitely unable) to 5 (definitely able).

\section{Statistical analysis}

The reliability of the measurements was assessed by means of a method of internal consistency using the formula of Cronbach's alpha. All alpha values were satisfactory to high: $\alpha=0.93$ for the perceived benefits of PA; $\alpha=0.87$ for the perceived barriers to PA; $\alpha=0.90$ for the perceived severity of diseases resulting from physical inactivity; $\alpha=0.81$ for the perceived threat of diseases resulting from physical inactivity; $\alpha=0.87$ for selfefficacy. The skewness values ranged from 0.22 to 1.17 , while kurtosis ranged from 0.06 to 1.57 , indicating that the measured variables were close to normal. The equality of variances was tested and confirmed by means of Levene's test. The differences between stages of change were analysed using the analysis of variance (ANOVA) with post-hoc comparisons performed using Tukey's test. After the reporting the p-value, measures of effect size were also computed: partial eta-squared in ANOVA and Cohen's $d$ in t-tests.

\section{Results}

Due to the low number of respondents in the precontemplation and contemplation stages, for the purpose of the study, they were combined into one stage labelled "inactive". The distribution of the respondents in the stages of change was as follows: $5.92 \%$ reported no PA at all (precontemplation and contemplation stages), 44.38\% reported irregular PA (preparation), $8.88 \%$ reported engaging in regular PA but for less than half a year (action stage), 34.91\% reported regular and habitual PA (maintenance stage), and 5.92\% reported having dropped out from regular PA (relapse or regression to inactivity). In respect 
Table 1. Results of ANOVA for each of the HBM variables in each stage of exercise change [means (SDs)]

\begin{tabular}{|c|c|c|c|c|c|c|c|c|}
\hline Variable & In & $\mathrm{Pr}$ & Ac & $\mathrm{Ma}$ & $\operatorname{Re}$ & ANOVA & Post hoc & $\eta^{2}$ \\
\hline Perceived benefits & $\begin{array}{c}3.09 \\
(0.26)\end{array}$ & $\begin{array}{c}3.31 \\
(0.40)\end{array}$ & $\begin{array}{c}3.54 \\
(0.40)\end{array}$ & $\begin{array}{c}3.50 \\
(0.53)\end{array}$ & $\begin{array}{c}3.61 \\
(0.48)\end{array}$ & $\begin{array}{c}F_{(4,160)} 3.54 \\
p=0.009\end{array}$ & In. $P r<A c . M a . R e$ & 0.08 \\
\hline Perceived barriers & $\begin{array}{c}2.72 \\
(0.87)\end{array}$ & $\begin{array}{c}2.55 \\
(0.89)\end{array}$ & $\begin{array}{c}2.49 \\
(0.94)\end{array}$ & $\begin{array}{c}1.86 \\
(0.78)\end{array}$ & $\begin{array}{c}2.77 \\
(1.36)\end{array}$ & $\begin{array}{c}\mathrm{F}_{(4,139)} 5.49 \\
\mathrm{p}<0.001\end{array}$ & In. $\operatorname{Pr} . A c>M a<R e$ & 0.14 \\
\hline Perceived severity & $\begin{array}{c}3.71 \\
(0.88)\end{array}$ & $\begin{array}{c}3.53 \\
(0.91)\end{array}$ & $\begin{array}{c}3.48 \\
(0.24)\end{array}$ & $\begin{array}{c}3.69 \\
(1.16)\end{array}$ & $\begin{array}{c}3.64 \\
(0.88)\end{array}$ & $\begin{array}{c}F_{(4,152)} 0.35 \\
p=0.842\end{array}$ & ns & 0.01 \\
\hline Perceived risk & $\begin{array}{c}2.75 \\
(0.80)\end{array}$ & $\begin{array}{c}2.50 \\
(0.93)\end{array}$ & $\begin{array}{c}2.70 \\
(0.95)\end{array}$ & $\begin{array}{c}2.41 \\
(0.77)\end{array}$ & $\begin{array}{c}3.32 \\
(0.76)\end{array}$ & $\begin{array}{c}F_{(4,152)} 2.25 \\
p=0.066\end{array}$ & Pr. $\mathrm{Ma}<\mathrm{Re}$ & 0.06 \\
\hline Self-efficacy & $\begin{array}{c}2.97 \\
(0.75)\end{array}$ & $\begin{array}{c}3.47 \\
(0.81)\end{array}$ & $\begin{array}{c}3.73 \\
(0.64)\end{array}$ & $\begin{array}{c}3.77 \\
(0.60)\end{array}$ & $\begin{array}{c}3.13 \\
(0.50)\end{array}$ & $\begin{array}{c}\mathrm{F}_{(4,149)} 4.17 \\
\mathrm{p}=0.003\end{array}$ & $\ln <\operatorname{Pr}<$ Ac. $M a>\operatorname{Re}$ & 0.10 \\
\hline
\end{tabular}

* In = inactive; $\operatorname{Pr}=$ preparation; Ac = Action; $\mathrm{Ma}=$ maintenance; $\mathrm{Re}=$ relapse.

to the HBM variables, two differences were observed: women, as compared to men, perceived physical activity as more beneficial to their health $(\mathrm{M}=3.44 \mathrm{vs} .3 .19, \mathrm{SD}=0.41$ vs. 0.66 , respectively; $\mathrm{t}_{(164)}=2.5 ; \mathrm{p}=0.001 ; \mathrm{d}=0.46$ ); however, women also evaluated the risk of being affected by hypokinetic diseases as higher $\left(\mathrm{M}=2.89\right.$ vs. $2.49, \mathrm{SD}=0.93$ vs. 0.85 , respectively; $\mathrm{t}_{(156)}=-2.12$; $\mathrm{p}=0.035 ; \mathrm{d}=0.45)$. In both cases, the size of the differences between both groups was small to modest.

MANOVA revealed a significant main effect for stages of exercise change for the Health Belief Model psychosocial variables (Wilks's lambda $\left.=0.708 ; \mathrm{F}_{(20,395.63)}=2.173 ; \mathrm{p}=0.003\right)$. Follow-up ANOVA showed that apart from perceived severity (insignificant) and perceived risk (a tendency toward significance: $F_{(4,152)}=2.25 ; \mathrm{p}=0.066$ ), all remaining psychosocial variables differed significantly depending on the stage of exercise change. However, the effect sizes were small to moderate. The highest proportion of the explained variance (14\%) in the stages of change was observed in the case of perceived barriers to PA, whereas the lowest one was observed in perceived severity and perceived risk. ANOVA results for each of the HBM variables as broken down into each stage of exercise change are presented in table 1.

As post-hoc analyses revealed, mean scores of self-efficacy increased rapidly from the inactive stage to the action stage, then they remained quite stable in the maintenance stage, but they decreased in the relapse stage. The levels of the perceived barriers were highest in the inactive stage, and dropped in the preparation, action, and maintenance stages; yet, differences among the inactive, preparation, and action stages were insignificant. In the relapse stage, a significant increase in perceived barriers was observed. The perceived benefits were lowest in the inactive stage and preparation stage, and both these stages differed significantly from the remaining ones, which in turn did not show any significant differences. Analogously, there were no significant differences in perceived severity, while in perceived risk only relapsers differed significantly from active persons.

\section{Discussion}

Although the Health Belief Model (HBM) constitutes one of the most widely used theoretical frameworks for understanding and promoting health behaviours, little is known about its usefulness with respect to explaining physical activity behaviour. The variables which have been studied the most extensively are variables that are "universal" for many theories, such as self efficacy and barriers to exercise $[15,16,17,18]$. The remaining constructs and/or models considered as a whole have rarely been studied in the context of PA. The existing body of knowledge suggests, however, that PA is not well predicted by the model, especially in young, healthy people who are not concerned with avoiding diseases $[3,19]$. The situation may look different in older people who should be more concerned with their health behaviour. Therefore, the purpose of the study was to assess how the HBM variables differ across the stages of readiness for physical activity in a sample of older adults studying at Universities of the Third Age.

The results of the study showed that the determinants of health behaviours predicted by the HBM, except perceived risk and perceived severity, did vary between the stages of the participants' readiness for PA. As far as perceived risk is concerned, a trend toward significance was observed, and post-hoc comparisons revealed that there was an increase of this variable in the relapse stage in comparison with the preparation and maintenance stages. In other words, individuals who exercised regularly in the past but, for some reason, quit PA were most prone to thinking of themselves as vulnerable to hypokinetic diseases; although, at the same time, they did not have a lifestyle that prevents these diseases.

Perceived severity was found to be non-predictive of the stages of readiness for PA. This finding is not in line with the findings of Courneya who discovered that the said variable distinguished older adults classified as being in the precontemplation stage from those in the contemplation, action, and maintenance stages, as well as those in the preparation stage from those in the action and maintenance stages [20]. According to this author, the main function of perceived severity of being inactive is to drive people to consider changing their lifestyle. Even those who are in the preparation stage and are somewhat active would be motivated to increase their physical activity if they believed that the consequences of being inactive are very severe [20].

As mentioned before, the remaining constructs distinguished people in particular stages of readiness for physical activity. The greatest magnitude of the relationship between the stages of readiness for physical activity was observed in perceived barriers to physical activity. The value of partial etasquared was 0.14 , which is interpreted as the lower end of a large effect size. Perceived barriers were lowest in the maintenance stage, which differed significantly from the remaining stages. 
What is interesting to note is that those who were active in the past but relapsed into inactivity perceived barriers in a similar way as those who had never exercised regularly.

In the case of perceived benefits and self-efficacy, the effect sizes were medium, accounting for $8 \%$ and $10 \%$ variance in stages of readiness. Perceived benefits were significantly lower in the inactive stage than in the action and maintenance stages and even the relapse stage. This means that, according to the results of the study, older adults who were active but have stopped engaging in this kind of activity still consider it as beneficial. On the other hand, people who have never been active do not see any reason to take up physical activity. Of course, the relationship between the stages of change and perceived benefits may be bidirectional. People who are unaware of the benefits of PA avoid it, seeing no reason to make the effort, but people who avoid PA will never have the chance to personally experience the benefits arising from regular exercise, and, therefore, those who are at higher stages of change are "benefits perceivers" because they can draw conclusions from their own experience. Last but not least, the variable of self-efficacy changed linearly across stages, except for relapse, in which rapid decrease was observed.

This study is unique in that it explored the HBM variables in relation to the PA of older adults, confirming doubts as to the usefulness of this model in explaining PA behaviour. These doubts have been expressed particularly with regard to the applicability of the model constructs in explaining the PA of young people who are rather not concerned about such issues as exposing themselves to the risk of diseases caused by their sedentary lifestyle or being physically active just because this kind of behaviour is beneficial. Older adults are more concerned with their health and more inclined to take actions seeing the reasons to do so; therefore, it could be expected that the HBM should be more predictive of their intentions and behaviours related to PA. The results of this study only partially support the above hypothesis. Our findings showed that the reasons why people undertake PA are related to such factors as the belief that a person is susceptible to diseases resulting from sedentary lifestyle; the facts that these diseases will have a harmful effect on their life and that a physically active lifestyle will prevent these diseases were rather weak predictors of stages of readiness for PA, with effect sizes from 0.01 to 0.08 . Therefore, interventions aimed at informing older adults about the benefits of PA and the threat of the consequences of sedentary lifestyle can be expected to have a rather small influence on their readiness for PA. Only two factors were moderately-to-strongly related to stages of change, namely perceived barriers and self-efficacy. This would support the findings from other studies and imply that strategies which increase self-efficacy in PA and enhance the ability to overcome barriers to PA should be undertaken.

Despite the strengths and the findings of this study, there are some limitations which should be highlighted. Firstly, its cross-sectional design does not allow for establishing unequivocal causal relationships. Secondly, the data were self-reported, and, therefore, the results can be biased due to socially desirable responding. Thirdly, the participants were recruited from Universities of the Third Age, and thus the sample might be overrepresented by people who are more conscious about health and factors affecting it. This could be the cause of the relatively low number of respondents who declared being in the precontemplation stage, which made the authors introduce the inactive stage that included people who were inactive and did not even want to change their lifestyle and people who were inactive but were considering changing their lifestyle. Finally, the study sam- ple consisted of substantially more females than males, and thus the results may be "biased" by the perceptions of the former.

\section{Conclusion}

The Health Belief Model weakly predicts increases in stages of readiness for physical activity in older adults. In practical terms, this means that interventions aimed at increasing the perceived severity of hypokinetic diseases, susceptibility to them, and perceived benefits of physical activity will most likely be ineffective in elderly people. However, our findings provide further support for the importance of self-efficacy and perceived barriers to physical activity. Thus, even in older adults, interventions should be targeted at promoting belief in one's capacity for performing exercise behaviours and managing common barriers to them.

\section{Literature}

1. Dishman R.K., Washburn R.A., Heath G.W. (2004). Physical activity epidemiology. Champaign: Human Kinetics Publishers.

2. Drygas W., Kwaśniewska M., Kaleta D., Pikala M., Bielecki W., Gluszek J. et al. (2009). Epidemiology of physical inactivity in Poland: Prevalence and determinants in a former communist country in socioeconomic transition. Public Health 123, 592-597.

3. Buckworth J., Dishman R.K. (2002). Exercise psychology. Champaign: Human Kinetics Publishers.

4. Sonstroem R.J. (1988). Psychological models. In R.K. Dishman (ed.), Exercise adherence. Its impact on public health (pp. 125-153). Champaign: Human Kinetics Publishers.

5. Daddario D.K. (2007). A review of the use of the Health Belief Model for weight management. MedSurg Nursing 16, 363-366.

6. Hanson J.A., Benedict J.A. (2002). Use of the Health Belief Model to examine older adults' food-handling behaviors. Journal of Nutrition Education and Behavior 34, S25-S30.

7. Deshpande S., Basil M.D., Basil D.Z. (2009). Factors influencing healthy eating habits among college students: An application of the Health Belief Model. Health Marketing Quarterly 26, 145-164.

8. Ross T.P., Ross L.T., Rahman A., Cataldo S. (2010). The bicycle helmet attitudes scale: Using the Health Belief Model to predict helmet use among undergraduates. Journal of American College Health 59, 29-36.

9. Greene K., Brinn L.S. (2003). Messages influencing college women's tanning bed use: Statistical versus narrative evidence format and a self-assessment to increase perceived susceptibility. Journal of Health Communication 8, 443-461.

10. Coe A.B., Gatewood S.B.S., Moczygemba L.R., Goode J.V., Beckner J.O. (2012). The use of the Health Belief Model to assess predictors of intent to receive the novel (2009) HINl influenza vaccine. Innovations in Pharmacy 3, 1-11.

11. Biddle S.J.H., Mutrie N. (2001). Psychology of physical activity. Determinants, well-being, and interventions. LondonNew York: Routledge.

12. Anshel M.H. (2014). Applied health fitness psychology. Champaign: Human Kinetics Publishers.

13. Prochaska J., Marcus B.H. (1994). The trans-theoretical model: Applications to exercise behavior. In R.K. Dishman (ed.), Advances in exercise adherence. Champaign: Human Kinetics Publishers. 
14. Marcus B.H., Rakowski W., Rossi J.S. (1992). Assessing motivational readiness and decision-making for exercise. $\mathrm{He}$ alth Psychology 11, 257-261.

15. Cardinal B.J. (1997). Predicting exercise behavior using components of the trans-theoretical model of behavior change. Journal of Sport Behavior 20, 272-284.

16. Kearney J.M., de Graaf C., Damkjaer S., Engstron L.M. (1992). Stages of change towards physical activity in a nationally representative sample in the European Union. Public Health Nutrition 2, 115-124.

17. Nishida Y., Suzuki H., Wang D.H., Kira S. (2003). Psychological determinants of physical activity in Japanese female employees. Journal of Occupational Health 45, 15-22.
18. Tai-Seale T. (2003). Stage of change specific triggers and barriers to moderate physical activity. American Journal of Health Behavior 2, 219-227.

19. Sas-Nowosielski K., Grabara M., Hadzik A. (2013). Health Belief Model variables as predictors of light, moderate and vigorous physical activity among young adults. New Education Review 32, 194-203.

20. Courneya K.S. (1995). Perceived severity of the consequences of physical inactivity across the stages of change in older adults. Journal of Sport E Exercise Psychology 17, 447-457.

Submitted: July 12, 2016

Accepted: September 16, 2016 